\title{
A software agent enabled biometric security algorithm for secure file access in consumer storage devices
}

Article

Accepted Version

Amin, R., Sherratt, R. S., Giri, D., Islam, S. K. H. and Khan, M. K. (2017) A software agent enabled biometric security algorithm for secure file access in consumer storage devices. IEEE Transactions on Consumer Electronics, 63 (1). pp. 5361. ISSN 0098-3063 doi:

https://doi.org/10.1109/TCE.2017.014735 Available at https://centaur.reading.ac.uk/69397/

It is advisable to refer to the publisher's version if you intend to cite from the work. See Guidance on citing.

Published version at: http://ieeexplore.ieee.org/document/7931970/

To link to this article DOI: http://dx.doi.org/10.1109/TCE.2017.014735

Publisher: IEEE

All outputs in CentAUR are protected by Intellectual Property Rights law, including copyright law. Copyright and IPR is retained by the creators or other copyright holders. Terms and conditions for use of this material are defined in the End User Agreement. 


\section{CentAUR}

Central Archive at the University of Reading

Reading's research outputs online 


\section{Full-Text version}

Title: $\quad$ A Software Agent Enabled Biometric Security Algorithm for Secure File Access in Consumer Storage Devices

Authors: Ruhul Amin,

Department of Computer Science and Engineering, Thapar University, Patiala, Punjab, India

(e-mail: amin_ruhul@live.com)

R. Simon Sherratt, Fellow, IEEE

Department of Biomedical Engineering, the University of Reading,

RG6 6AY, UK

(e-mail: sherratt@ieee.org)

Debasis Giri

Department of Computer Science and Engineering, Haldia Institute of Technology,

Haldia-721657, India

(e-mail: debasis_giri@hotmail.com)

SK Hafizul Islam

Department of Computer Science and Engineering, Indian Institute of Information Technology,

Kalyani, West Bengal 741235, India

(e-mail: hafi786@gmail.com)

Muhammad Khurram Khan, Senior Member, IEEE

Center of Excellence in Information Assurance (CoEIA), King Saud University, Riyadh 11451, Saudi Arabia

(e-mail: mkhurram@ksu.edu.sa)

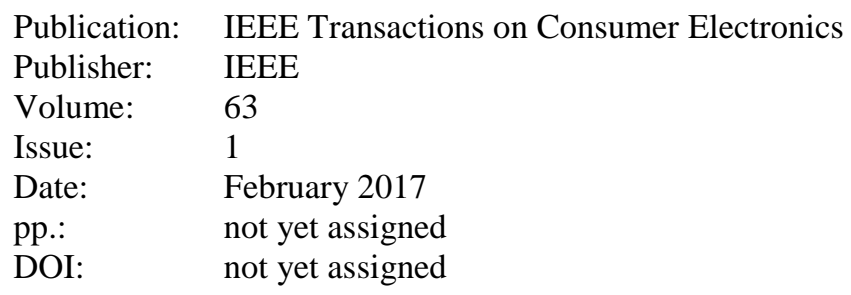

\section{Abstract}

In order to resist unauthorized access, consumer storage devices are typically protected using a low entropy password. However, storage devices are not fully protected against an adversary because the adversary can utilize an off-line dictionary attack to find the correct password and/or run an existing algorithm for resetting the existing password. In addition, a password protected device may also be stolen or misplaced allowing an adversary to easily retrieve all the stored confidential information from a removable storage device. In order to protect the consumer's confidential information that has been stored, this paper proposes a mutual authentication and key negotiation protocol that can be used to protect the confidential information in the device. The functionality of the protocol enables the storage device to be secure against relevant security attacks. A formal security analysis using Burrows-Abadi-Needham (BAN) logic is presented to verify the presented algorithm. In addition, a performance analysis of the proposed protocol reveals a significantly reduced communication overhead compared to the relevant literature.

Index Terms

Security Protocol, Biometric, Computer System, BAN logic, File Secrecy 


\section{INTRODUCTION}

Consumer storage is commonly used to store and retrieve digital information. Consumers often store confidential information, files, or digital media purchases in the device. These devices are low cost and easily portable so the consumer often carries the device when travelling. As a result, the device may be lost or stolen by an adversary. If the confidential information is not protected, an adversary can easily retrieve the stored information from the device memory. However, the adversary faces a problem to retrieve the information from the store if the device is password protected. It is worth noting that a user's password (typically low entropy) cannot provide a strong secure system under a cryptographic dictionary attack. Indeed, many techniques are currently available to guess the password to access the device.

Mutual authentication and key agreement protocols are a popular paradigm in client-server environments to prevent unauthorized access. In 1981, Lamport [1] first introduced the secure communication client-server architecture and then numerous protocols [2]-[4] have been proposed for several applications, including wireless sensor networks [5], medical systems [6] and file security for USB based Mass Storage Devices (USB MSD) [7]-[12]. In order to provide secure access, authentication protocols play an important role.

Significant literature is now available to provide solutions to protect confidential files stored in a USB MSD. Yang et al. [7] first proposed a secure authentication protocol using the Schnorr Signature to protect the information stored. However, Chen et al. [8] argued that the protocol from Yang et al. [7] was not secure against the forgery attack and the replay attack. Furthermore, Lee et al. [9] argued that the protocol by Chen et al. [8] was computationally inefficient. In order to solve the security weaknesses, Lee et al. [9] proposed the three-factor authentication protocol based on elliptic curve cryptography. The protocol from Lee et al. [9] required the user's password, biometric and smartcard information as authentication tokens. More recently, He et al. [10] demonstrated that the protocol proposed by Lee et al. [9] was not secure against the password guessing attack, Denial-of-Service (DoS) attack and the replay attack, so proposed an improved three-factor authentication scheme. In order to resist the DoS attack, He et al. [10] employed the concept of the fuzzy extractor [13], [14]. In 2015, Amin and Biswas [15] proposed a three-factor authentication protocol for the same environment using a hash function to achieve a lower computation cost than existing protocols [9], [10].

This paper proposes a mutual authentication and key agreement protocol to provide only authorized access to confidential information stored on the device with the aid of a Registration Server (RS). A new user completes a registration procedure with RS allowing RS to deliver a link via e-mail from which the user can download and install registration software in their device which also incorporates the required secure access information relevant for only each user. In order to provide secure access to files, the user provides the necessary identity, password and biometric information. The device checks the legitimacy of the user and then negotiates a session key with RS. It is to be noted that this session key is used to encrypt the files in the storage device.

The rest of the paper is organized as follows: Section II presents an overview of the contribution and the novelty claims. Section III presents the hash function, fuzzy extractor and elliptic curve cryptography. The proposed protocol is provided in Section IV.

The security analysis using BAN logic is discussed in Section V. Section VI provides the performance evaluation and comparison of the proposed protocol with related protocols. Section VII concludes the paper. TABLE I shows the nomenclature that is used throughout the paper. 
TABLE I

NOMENCLATURE

\begin{tabular}{ll}
\hline \hline Term & Usage \\
\hline$U_{i}$ & No-th user \\
$R S$ & Remote server \\
$P W_{i}$ & Password of user $U_{i}$ \\
$B T_{i}$ & Biometric Template of user $U_{i}$ \\
$I D_{i}$ & Identity of user $U_{i}$ \\
$E_{k}[]$ & Symmetric key encryption using key $k$ \\
$D_{k}[]$ & Symmetric key decryption using key $k$ \\
$x$ & Secret key of the remote server \\
$\left(P_{x}, P_{y}\right)$ & x and $y$ coordinate of the elliptic curve point $P$ \\
$T_{i}$ & Current timestamp of $U_{i}$ 's storage device \\
$T_{j}$ & Current timestamp of the Remote server \\
$\Delta T$ & Estimated time delay \\
$\mathrm{UNSID}$ & Unique software identity \\
$\mathrm{SL}$ & Software link \\
$h(\cdot)$ & Cryptographic one-way hash function \\
$R E P()$ & $R E P$ procedure in fuzzy extractor \\
$G E N()$ & GEN procedure in fuzzy extractor \\
$\bigoplus$ & Bitwise XOR operator \\
$\|$ & Concatenation operator \\
$(a . b)$ & Point multiplication operation of $a$ and $b$ \\
\hline
\end{tabular}

\section{SYSTEM ENVIRONMENT}

In this work, a Registration Server (RS) delivers a link to all the users who have performed registration successfully, and then each user uses the link to obtain and install software in their device while also providing their credentials (password, identity and biometric signature.) Note that while the password may be guessed, it is hard to guess biometric signatures. Then, the software encrypts important files by using a negotiated key to provide security on the storage file. Whenever, the user of that device wants to access that file, RS first verifies the user and then provides a decryption key to recover the original file. All the files are then encrypted using a new session key. However, we argue that a storage device will still not be completely security protected. Hence, we have devised a standard security protocol which protects the storage device to defend unauthorized access. Firstly we have used the concept of biometric data along with a password in our protocol, hence it is difficult to guess the password along with biometric information. Secondly, an attacker cannot utilize a resetting technique, as we have mentioned in our protocol that if the attacker desires to use resetting technique, he/she first has to login into the system. As the attacker cannot login into the system without biometric data, the resetting technique is not usable.

This paper achieves the following contributions:

$\checkmark$ A mutual authentication and key negotiation protocol to provide security protection of the stored information on the storage device,

$\checkmark$ Security analysis to show that the proposed protocol is robust against known security attacks. Furthermore, in the proposed scheme, the mutual authentication and session key agreement have been verified using BAN logic.

$\checkmark$ Significantly less communication overhead and computation costs than other related systems.

\section{PRELIMINARIES}

This section defines the fuzzy extractor [10]-[14] and the hash function [15] to analyze the security of the proposed protocol. Furthermore, the hardness assumption on the elliptic curve group is discussed.

Definition 1: A cryptographic one-way hash function maps a binary string of an arbitrary length to a binary string of fixed length, called the hashed value. It can be symbolized as: $h:\{0,1\}^{*} \rightarrow\{0,1\}^{n}$, where $n$ is a positive integer. The properties of the hash function have been presented [4], [5].

Definition 2: A fuzzy system based collision resistant extractor can be modeled as a procedure which takes a binary string, say $b$, of some metric space $M \in\{0,1\}^{n}$ as an input for some positive number $n$ and outputs a random string, say $\psi \in\{0,1\}^{l}$ for some 
positive number $l$ and an auxiliary string, say $\theta \in\{0,1\}^{r}$ for some positive number $r$, where $r$ can be $l$ or $n$. This mapping procedure is denoted by GEN: $M \rightarrow \psi \times \theta$. Another procedure which takes two inputs: (i) a binary string say, $b^{\prime}$ of the metric space $M \in\{0,1\}^{n}$, where $\left(b \neq b^{\prime}\right)$, and (ii) an uniform distribution binary string say, $\theta \in\{0,1\}^{r}$, and it produces the random string $\psi \in\{0,1\}^{l}$ as output. This mapping procedure is denoted by $R E P: M \times \theta^{\prime} \rightarrow \psi$.

\section{A. Elliptic Curve Cryptography (ECC)}

The concept of elliptic curve cryptography was introduced by Kobiltz [16] and Miller [17], to design public key cryptosystems. Let $E_{p}(a, b)$ be a set of elliptic curve points over prime field $F_{p}$, where $\mathrm{p}$ is a large prime number. The elliptic curve equation is defined as: $y^{2}=x^{3}+a x+b \bmod p$ with $(a, b) \in F_{p}$ and $\left(4 a^{3}+27 b^{2}\right) \bmod p \neq 0$. The additive ECC group is defined as: $G_{p}=\left\{(x, y): x, y \in F_{p}\right.$ and $\left.(x, y) \in E_{p}(a, b)\right\} \cup\{O\}$, where the point $O$ is known as the 'Point at Infinity'. The scalar point multiplication on the cyclic group $G_{p}$ is defined as: $\left.[k] \cdot P=P+P+\ldots+P\right)$, that means $k$ times addition of $P$.

Definition 3: Elliptic curve discrete logarithm problem: Given $\left(Q, R \in G_{p}\right)$, computation of the integer $k \in Z_{p} *$ is hard, where $R=[k] \cdot Q$.

Definition 4: Elliptic curve computational Diffie-Helman problem: Given $(P,[a] . P,[b] . P)$, for some $a, b \in Z_{p}{ }^{*}$, computation of $[a] \cdot[b] . P$ is hard.

\section{PROPOSED PROTOCOL}

This section describes the proposed mutual authentication and key negotiation protocol, which includes seven phases, (A) Registration and software installation phase, (2) Login phase, (C) Mutual authentication and key negotiation phase, (D) File management phase, (E) File accessing phase, (F) Password renewal phase and (G) Biometric renewal phase.

Initially, RS chooses a secret key $x$ and computes $P_{p u b}=[x] . P$ as the corresponding public key. It should be noted that execution of the registration phase and the registration software installation phase is performed only once.

\section{A. Registration and Software Installation Phase}

Initially, each new user $U_{i}$ must complete a registration procedure with RS. In this phase, $U_{i}$ provides their information securely or in person (off-line mode) to RS. Then, RS securely sends to $U_{i}$, via e-mail, a link to downloadable registration software which must be installed in the storage device. The description of this phase is given below:

Step 1: $U_{i}$ first chooses $\left\langle I D_{i}, P W_{i}\right\rangle$ and scans the user's biometric template, $B T_{i}$, such as a fingerprint. This work uses the biometric template to provide a high degree security since biometric templates cannot easily be forged [10]-[15]. $U_{i}$ 's device computes $P W B_{i}=h\left(P W_{i} \| b_{i}\right)$, where $b_{i}$ is a random number generated by $U_{i}$ and then sends $\left\langle I D_{i}, P W B_{i}, B T_{i}\right\rangle$ and a valid email address to RS securely either using Transport Layer Security (TLS) or in person (off-line mode.)

Step 2: After receiving the registration message, RS computes $\left(\psi_{i}, \theta_{i}\right)=G E N\left(B T_{i}\right), A_{i}=h\left(P W B_{i} \| \psi_{i}\right), G_{i}=h\left(I D_{i} \| x\right)$, $B_{i}=G_{i} \oplus P W B_{i}, C_{i}=\theta_{i} \oplus h\left(I D_{i} \| P W B_{i}\right)$ and $D_{i}=E_{G_{i}}\left(A_{i}\left\|B_{i}\right\| C_{i}\right)$, where $G E N()$ is the fuzzy extractor function.

Step 3: RS then embeds $\left\langle D_{i}, I D_{i}, B_{i}, G E N(), R E P(), h()\right\rangle$ into the required registration software including all necessary parameters for the ECC cryptosystem. The registration software is a simple software application that must be installed in the consumer device. RS needs to maintain a database for storing all the registration information for all the consumers. RS stores 
$\left(I D_{i}, U N S I D_{i}, S L_{i}, \Xi\right)$ into the database, where $U N S I D_{i}$ and $S L_{i}$ are the unique software identity and software link respectively, and $\Xi$ indicates empty attributes used to store the encrypted key. Finally, RS delivers to $U_{i}$ via e-mail a link to user specific registration software (that includes $S L_{i}$.) This registration software is provided by the registration server to all the consumers with the software content varying with the user.

Step 4: After receiving the link for $U_{i}$ to download the registration software, $U_{i}$ installs it on their personal storage device. $U_{i}$ then inputs $b_{i}$ into the registration software. Finally, the registration software installed in $U_{i}$ 's storage device contains $\left\langle D_{i}, I D_{i}, B_{i}, b_{i}, G E N(), R E P(), h()\right\rangle$.

\section{B. Login Phase}

This phase ensures that a non-registered user could not install the registration software without providing the correct information. The device runs the registration software now installed in the storage device and the software requests $U_{i}$ to input their identity, password and biometric information $\left(I D_{i}, P W_{i}\right.$ and $\left.B T_{i}\right)$. Then the registration software checks the legitimacy of $U_{i}$ by verifying the user's information by calculating $P W B_{i}^{\prime}=h\left(P W_{i} \| b_{i}\right), \quad G_{i}^{\prime}=B_{i} \oplus P W B_{i}{ }^{\prime},\left(A_{i}{ }^{\prime}\left\|B_{i}{ }^{\prime}\right\| C_{i}{ }^{\prime}\right)=D_{G_{i}^{\prime}}\left(D_{i}\right)$, $\theta_{i}^{\prime}=h\left(I D_{i} \| P W B_{i}^{\prime}\right) \oplus C_{i}{ }^{\prime}, \psi_{i}{ }^{\prime}=\operatorname{REP}\left(B_{i}, \theta_{i}^{\prime}\right)$ and $A_{i}^{\prime \prime}=h\left(P W B_{i}^{\prime} \| \psi_{i}^{\prime}\right)$. The registration software checks whether the conditions $A_{i}{ }^{\prime \prime}=? A_{i}{ }^{\prime}$ and $B_{i}{ }^{\prime}=? B_{i}$ holds. If both the conditions are true, then the registration software of $U_{i}$ accepts that the information provided by $U_{i}$ is correct; otherwise, it aborts the session.

\section{Mutual Authentication and Key Negotiation Phase}

This phase first achieves mutual authentication and then negotiates a session key between the registration software of $U_{i}$ and RS over an insecure channel. In this process, $U_{i}$ and RS perform the following steps:

Step 1: $U_{i}$ runs the registration software installed in his/her device and then provides their $I D_{i}, P W_{i}$ and $B T_{i}$ to the registration software. Then the registration software of $U_{i}$ computes $P W B_{i}^{\prime}=h\left(P W_{i} \| b_{i}\right), G_{i}^{\prime}=B_{i} \oplus P W B_{i}^{\prime}, \quad\left(A_{i}{ }^{\prime}\left\|B_{i}{ }^{\prime}\right\| C_{i}{ }^{\prime}\right)=D_{G_{i}^{\prime}}\left(D_{i}\right)$, $\theta_{i}^{\prime}=h\left(I D_{i} \| P W B_{i}^{\prime}\right) \oplus C_{i}{ }^{\prime}, \quad \psi_{i}^{\prime}=\operatorname{REP}\left(B_{i}, \theta_{i}^{\prime}\right)$ and $A_{i}^{\prime \prime}=h\left(P W B_{i}^{\prime} \| \psi_{i}^{\prime}\right)$. The registration software in $U i$ 's device checks conditions $A_{i}{ }^{\prime \prime}=$ ? $A_{i}{ }^{\prime}$ and $B_{i}{ }^{\prime}=? B_{i}$. If both the conditions are not correct, registration software of $U_{i}$ aborts the connection; otherwise, accepts $U_{i}$.

Step 2: The registration software in $U_{i}$ generates random number $r_{i}$ and sends $\left\langle I D_{i}, M_{5}, T_{i}\right\rangle$ to RS through an insecure channel, where $\quad M_{1}=\left[r_{i}\right] . P, \quad M_{2}=\left[\theta_{i}\right] \cdot M_{1}, \quad M_{3}=\left(K_{x}, K_{y}\right)=\left[G_{i}^{\prime}\right] \cdot P_{p u b}, \quad M_{4}=h\left(I D_{i}\left\|M_{1}\right\| M_{2}\left\|T_{i}\right\| K_{y}\right) \quad$ and $M_{5}=E_{K_{x}}\left(M_{1}\left\|M_{4}\right\| P W B_{i} \| C_{i}\right)$.

Step 3: After receiving $\left\langle I D_{i}, M_{5}, T_{i}\right\rangle$, RS first checks the existence of $I D_{i}$ in the user database held by RS. If the entry does not exist then RS rejects the connection, otherwise RS checks the timestamp validity condition $\left|T_{j}-T_{i}\right| \leq \Delta T$ holds, where $T_{j}$ is the current timestamp of RS. If it does not hold, RS rejects the connection; otherwise RS computes the legitimacy of $U_{i}$ by computing $G_{i}^{\prime}=h\left(I D_{i} \| x\right)$,

$$
M_{3}^{\prime}=\left(K_{x}^{\prime}, K_{y}^{\prime}\right)=\left[G_{i}^{\prime}\right] \cdot P_{p u b},\left(M_{1}\left\|M_{4}\right\| P W B_{i} \| C_{i}\right)=D_{K_{x}^{\prime}}\left(M_{5}\right), \theta_{i}^{\prime}=h\left(I D_{i} \| P W B_{i}\right) \oplus C_{i}, M_{2}^{\prime}=\left[\theta_{i}^{\prime}\right] \cdot M_{1} \text { and }
$$

$M_{3}{ }^{\prime}=h\left(I D_{i}\left\|M_{1}\right\| M_{2}{ }^{\prime}\left\|T_{i}\right\| K_{y}{ }^{\prime}\right)$. RS checks whether $M_{3}{ }^{\prime}=? M_{3}$ is true. If it is correct, then RS accepts $U_{i}$; otherwise, rejects $U_{i}$.

Step 4: RS generates random number $r_{j}$ and computes $S K_{j}=\left[r_{j}\right] . M_{2}^{\prime}, \quad M_{6}=h\left(I D_{i}\left\|P W B_{i}\right\| K_{x}^{\prime}\left\|r_{j}\right\| T_{j}\right) \quad$ and $M_{7}=E_{K_{y}^{\prime}}\left(M_{6} \| r_{j}\right)$. RS sends $M_{7}$ to the registration software in $U_{i}$ through a public channel. 
Step 5: After receiving $M_{7}$, the registration software in $U_{i}$ first checks whether the timestamp validity condition $\left|T_{j c}-T_{j}\right| \leq \Delta T$ holds, where $T_{j c}$ is the current timestamp at the user end. If it fails, the registration software of $U_{i}$ terminates the session; otherwise, it decrypts $M_{7}$ to obtain $\left(M_{6}, r_{j}\right)$ as $\left(M_{6} \| r_{j}\right)=D_{K_{y}^{\prime}}\left(M_{7}\right)$. The registration software in $U_{i}$ further computes $M_{6}^{\prime}=h\left(I D_{i}\left\|P W B_{i}\right\| K_{x}^{\prime}\left\|r_{j}\right\| T_{j}\right)$ and checks $M_{6}=M_{6}{ }^{\prime}$. If true, RS is verified. Then registration software in $U_{i}$ computes session key as $S K_{i}=\left[r_{j}\right] \cdot M_{2}$, which must be equal to $S K_{j}$ and used to encrypt desired files stored in the memory of the consumer storage device.

\section{File Management Phase}

After performing mutual authentication and key negotiation, the registration software can encrypt any chosen files $\left(F_{1}, F_{2}, \ldots\right.$, $F_{n}$ ), using the encryption key $S K_{i}$ for security protection. Note that, the registration software in $U_{i}$ can forget the encryption key after encrypting any files and send a confirmation message to RS. In this proposed protocol, RS maintains a table against each user $U_{i}$ with the identity $I D_{i}$. Now, RS stores $\left(S K_{i} \oplus h\left(I D_{i} \oplus x\right)\right)$ in the table against the identity $I D_{i}$.

\section{E. File Accessing Phase}

In this phase, $U_{i}$ makes a request to RS to access the encrypted files stored in the consumer's storage device. In order to do it, $U_{i}$ executes Steps 1-3 of the mutual authentication and key negotiation phase to verify the legitimacy of $U_{i}$ and generate a new session key. After the verification, RS first generates a random number $r_{j}{ }^{\prime}\left(r_{j}^{\prime} \neq r_{j}\right)$ and then computes the new session key $S K_{j}^{\prime}=\left[r_{j}\right] \cdot M_{2}^{\prime}$, where $\left(S K_{j}^{\prime} \neq S K_{j}\right)$ and the random numbers are different in each session. Furthermore, RS then computes $M_{6}=h\left(I D_{i}\left\|P W B_{i}\right\| K_{x}^{\prime}\left\|r_{j}^{\prime}\right\| T_{j}\right), M_{7}=E_{K_{y}^{\prime}}\left(M_{6} \| r_{j}^{\prime}\right)$ and retrieves $\left(S K_{i} \oplus h\left(I D_{i} \oplus x\right)\right)$ from the local table in RS and then computes the old session key $S K_{i}$. Finally, RS computes $M_{8}=E_{K_{x}^{\prime}}\left(S K_{i}\right)$ and sends $\left\langle M_{7}, M_{8}\right\rangle$ to $U_{i}$ through an insecure channel. Then, the registration software in $U_{i}$ decrypts $M_{7}$ and $M_{8}$ using $K_{y}{ }^{\prime}$ and $K_{x}{ }^{\prime}$ respectively. In order to verify the legitimacy of RS, the registration software in $U_{i}$ computes $M_{6}^{\prime}=h\left(I D_{i}\left\|P W B_{i}\right\| K_{x}^{\prime}\left\|r_{j}^{\prime}\right\| T_{j}\right)$. If $M_{6}^{\prime} \neq M_{6}$, the registration software of $U_{i}$ rejects the connection; otherwise, decrypts the encrypted files using the old key $S K_{i}$ obtained from $M_{8}$ and can then access the files. After that, the registration software in $U_{i}$ encrypts all the required files using the new key $S K_{i}^{\prime}=S K_{j}^{\prime}=\left[r_{j}^{\prime}\right] \cdot M_{2}$. Finally, the registration software in $U_{i}$ sends a confirmation message to RS that the obtained encrypted file is correct. Next, RS stores $\left(S K_{i}^{\prime} \oplus h\left(I D_{i} \oplus x\right)\right)$ in the table against $I D_{i}$.

\section{F. Password Renewal Phase}

This phase is infrequently used and the choice is dependent on the needs of the user. The description of the password update procedure is given as follows:

Step 1: $U_{i}$ runs the registration software installed in their device, then provides their $I D_{i}$, the current $P W_{i}$ and $B T_{i}$. Then the $U_{i}$ registration software computes $P W B_{i}^{\prime}=h\left(P W_{i} \| b_{i}\right), G_{i}{ }^{\prime}=B_{i} \oplus P W B_{i}{ }^{\prime},\left(A_{i}{ }^{\prime}\left\|B_{i}{ }^{\prime}\right\| C_{i}{ }^{\prime}\right)=D_{G_{i}^{\prime}}\left(D_{i}\right), \theta_{i}^{\prime}=h\left(I D_{i} \| P W B_{i}{ }^{\prime}\right) \oplus C_{i}{ }^{\prime}$, $\psi_{i}^{\prime}=\operatorname{REP}\left(B_{i}, \theta_{i}^{\prime}\right)$ and $A_{i}^{\prime \prime}=h\left(P W B_{i}{ }^{\prime} \| \psi_{i}{ }^{\prime}\right)$. The registration software in $U_{i}$ checks whether both $A_{i}{ }^{\prime \prime}=? A_{i}{ }^{\prime}$ and $B_{i}{ }^{\prime}=? B_{i}$ hold. If fasle $U_{i}$ aborts the session.

Step 2: $U_{i}$ inputs a new password $P W_{i}{ }^{*}$. The registration software in $U_{i}$ computes $P W B_{i}^{*}=h\left(P W_{i}^{*} \| b_{i}\right), B_{i}{ }^{*}=G_{i}{ }^{\prime} \oplus P W B_{i}{ }^{*}$, $A_{i}^{*}=h\left(P W B_{i}^{*} \| \psi_{i}^{\prime}\right), C_{i}^{*}=\theta_{i}^{\prime} \oplus h\left(I D_{i} \| P W B_{i}^{*}\right)$ and $D_{i}^{*}=E_{G_{i}^{\prime}}\left(A_{i}^{*}\left\|B_{i}^{*}\right\| C_{i}^{*}\right)$. 
Step 3: Finally, the registration software in $U_{i}$ replaces $D_{i}$ with new value $D_{i}{ }^{*}$ and keeps the remaining information unchanged. Thus, $U_{i}$ can change their old password without requesting any assistance from RS.

\section{G. Biometric Renewal Phase}

The execution of this phase is important whenever an existing user is willing to update their biometric information. The description of this phase is given as follows:

Step 1: $U_{i}$ runs the registration software installed the device and then provides previous login information $I D_{i}, P W_{i}$ and $B T_{i}$ to the registration software. Then the registration software in $U_{i}$ computes $P W B_{i}^{\prime}=h\left(P W_{i} \| b_{i}\right), \quad G_{i}^{\prime}=B_{i} \oplus P W B_{i}^{\prime}$, $\left(A_{i}{ }^{\prime}\left\|B_{i}{ }^{\prime}\right\| C_{i}{ }^{\prime}\right)=D_{G_{i}^{\prime}}\left(D_{i}\right), \theta_{i}^{\prime}=h\left(I D_{i} \| P W B_{i}^{\prime}\right) \oplus C_{i}{ }^{\prime}, \psi_{i}^{\prime}=R E P\left(B_{i}, \theta_{i}^{\prime}\right)$ and $A_{i}^{\prime \prime}=h\left(P W B_{i}^{\prime} \| \psi_{i}^{\prime}\right)$. The registration software in $U_{i}$ checks that both conditions $A_{i}{ }^{\prime \prime}=? A_{i}{ }^{\prime}$ and $B_{i}{ }^{\prime}=? B_{i}$. If false, the registration software in $U_{i}$ aborts the session.

Step 2: $U_{i}$ inputs new the biometric table $B T_{i}^{*}$. the registration software of $U_{i}$ computes $\left(\psi_{i}^{*}, \theta_{i}^{*}\right)=G E N\left(B T_{i}^{*}\right)$, $A_{i}^{*}=h\left(P W B_{i} \| \psi_{i}^{*}\right), C_{i}^{*}=\theta_{i}^{*} \oplus h\left(I D_{i} \| P W B_{i}\right)$, and $D_{i}^{*}=E_{G_{i}}\left(A_{i}^{*}\left\|B_{i}\right\| C_{i}^{*}\right)$.

Step 3: Finally, the registration software in $U_{i}$ replaces $D_{i}$ with the new value $D_{i}{ }^{*}$ and keeps the remaining information unchanged. Thus, $U_{i}$ can change/renew biometric information without requesting any assistance from RS.

\section{SECURITY ANALYSIS}

This section explores the security of the proposed mutual authentication and key negotiation protocol. This work employs BAN logic [5], [10], [18], [19] to demonstrate that the proposed protocol provides secure authentication. The informal security analysis examines that the proposed protocol is secure against relevant security attacks.

\section{A. Authentication Proof based on BAN Logic}

In this section, the security of the proposed protocol is analyzed using BAN logic. BAN logic is a well-known security verification and analysis model. It has been widely used for analyzing the security of authentication and session key agreement protocols. Some preliminaries and notations of BAN logic:

a) Principals are those agents involved in the protocol (usually people or programs).

b) Keys are used to encrypt messages symmetrically.

c) Public Keys are similar to keys except that they are used in pairs.

d) Nonces are message parts that are not meant to be repeated.

e) Timestamps are similar to nonce in that they are unlikely to be repeated.

Relevant BAN logic statements that are useful for analyzing security of the proposed protocol are:

$\mathrm{R} 1: \mathrm{P} \mid \equiv \mathrm{X}$ : $\mathrm{P}$ believes $\mathrm{X}$ or $\mathrm{P}$ would be entitled to believe $\mathrm{X}$. In particular, $\mathrm{P}$ can take $\mathrm{X}$ as true

$\mathrm{R} 2: \mathrm{P} \triangleleft \mathrm{X}$ : $\mathrm{P}$ sees $\mathrm{X}$. $\mathrm{P}$ has received some message $\mathrm{X}$ and is capable of reading and repeating it.

R3: $\mathrm{P} \mid \sim \mathrm{X}$ : $\mathrm{P}$ once said $\mathrm{X}$. $\mathrm{P}$ at some time sent a message including the statement $\mathrm{X}$. It is not known whether this is a replay, though it is known that $\mathrm{P}$ believed $\mathrm{X}$ when it was sent.

$\mathrm{R} 4: \mathrm{P} \Rightarrow \mathrm{X}$ : $\mathrm{P}$ has jurisdiction over $\mathrm{X}$. The principal $\mathrm{P}$ is an authority on $\mathrm{X}$ and should be trusted on this matter.

R5: $\#(X)$ : The message $X$ is fresh.

R6: $(\mathrm{X}, \mathrm{Y})$ : The formulae $\mathrm{X}$ or $\mathrm{Y}$ is one part of the formulae $(\mathrm{X}, \mathrm{Y})$.

R7: $\langle\mathrm{X}\rangle_{\mathrm{Y}}$ : The formulae $\mathrm{X}$ combined with the formulae $\mathrm{Y}$.

R8: $\{X\}_{K}$ : The formulae $X$ is encrypted under the formulae $\mathrm{K}$.

R9: $(\mathrm{X})_{\mathrm{K}}$ : The formulae $\mathrm{X}$ is hashed with the key $\mathrm{K}$.

R10: $\mathrm{P} \stackrel{K}{\longleftrightarrow}$ Q: Principal P and Q communicate via shared key K.

$\mathrm{R} 11: \mathrm{P} \Leftrightarrow \mathrm{Q}$ : The formulae $\mathrm{X}$ is a secret known only to $\mathrm{P}$ and $\mathrm{Q}$ only and possible to principal trusted by them. 
R12: SK: The session key used in the current session.

Relevant logical postulates of BAN logic for this work are:

- The message-meaning rule: $\frac{P \stackrel{K}{\longleftrightarrow} Q, P \triangleleft X}{P|\equiv Q| \sim X}$,

if the principal $\mathrm{P}$ believes that the secret key $\mathrm{K}$ is shared with the principal $\mathrm{Q}$ and $\mathrm{P}$ receives the message $\mathrm{X}$ encrypted with $\mathrm{K}$ then, $\mathrm{P}$ believes that the principal Q once sent the message $\mathrm{X}$.

- The freshness-conjuncatenation rule: $\frac{P \mid \equiv \neq(X)}{P \mid \equiv \neq(X, Y)}$,

if the principal believes that $\mathrm{X}$ is fresh, then the principal $\mathrm{P}$ believes freshness of $(\mathrm{X}, \mathrm{Y})$.

- The belief rule: $\frac{P|\equiv(X), P| \equiv(Y)}{P \mid \equiv(X, Y)}$,

if the principal $\mathrm{P}$ believes $\mathrm{X}$ and $\mathrm{Y}$, then the principal $\mathrm{P}$ believes $(\mathrm{X}, \mathrm{Y})$.

- The nonce verification rule: $\frac{P|\equiv \neq(X), P| \equiv Q \mid \sim X}{P|\equiv Q| \equiv X}$,

if the principal $\mathrm{P}$ believes that $\mathrm{X}$ is fresh and the principal $\mathrm{Q}$ once sent $\mathrm{X}$ then, principal $\mathrm{P}$ believes that $\mathrm{Q}$ believes $\mathrm{X}$.

- The jurisdiction rule: $\frac{P|\equiv Q \Rightarrow X, P| \equiv Q \mid \equiv X}{P \mid \equiv X}$,

if the principal believes that $\mathrm{Q}$ has jurisdiction over $\mathrm{X}$ and $\mathrm{Q}$ believes $\mathrm{X}$, then $\mathrm{P}$ believes that $\mathrm{X}$ is true.

- The session key rule: $\frac{P|\equiv \neq(X), P| \equiv Q \mid \equiv(X)}{P \mid \equiv P \stackrel{K}{\longleftrightarrow} Q}$,

if the principal $\mathrm{P}$ believes that the session key is fresh and the principal $\mathrm{P}$ and $\mathrm{Q}$ believes $\mathrm{X}$, which are the necessary parameters of the session key, then principal $\mathrm{P}$ believes that he/she shares the session key $\mathrm{K}$ with $\mathrm{Q}$.

In order to prove the proposed protocol secure, the proposed protocol must satisfy the following goals based on BAN logic, where $\mathrm{RS}$ and $U_{i}$ define registration server and consumer respectively.

Goal 1: $U_{i} \equiv U_{i} \stackrel{S K}{\longrightarrow} R S$

Goal 2: $U_{i}|\equiv R S| U_{i} \stackrel{S K}{\longrightarrow} R S$

Goal 3: $R S \mid \equiv R S \stackrel{S K}{\longrightarrow} U_{i}$

Goal 4: $R S \equiv U_{i} \equiv R S \stackrel{S K}{\longrightarrow} U_{i}$

The proposed protocol is transformed to the idealized form as:

$$
\begin{aligned}
& M S G_{1}: U_{i} \rightarrow R S:\left\langle I D_{i}, M_{5}, T_{i}\right\rangle:\left\langle M_{1}\right\rangle_{G_{i}} \\
& M S G_{2}: R S \rightarrow U_{i}:\left\langle M_{7}\right\rangle:\left\langle r_{j}\right\rangle_{K_{x}}
\end{aligned}
$$

The following assumptions about the initial state of the protocol are given:

$$
\begin{aligned}
& A S M_{1}: U_{i} \equiv \neq\left(r_{i}, r_{j}\right) \\
& A S M_{2}: R S \equiv\left(r_{j}, r_{i}\right) \\
& A S M_{3}: U_{i} \equiv U_{i} \stackrel{G_{i}}{\longrightarrow} R S \\
& A S M_{4}: R S \equiv R S \stackrel{K x}{\longrightarrow} U_{i} \\
& A S M_{5}: U_{i} \equiv R S \Rightarrow r_{j}
\end{aligned}
$$


$A S M_{6}: R S \mid U_{i} \Rightarrow r_{i}$

Applying BAN logic rules and assumptions:

$M S G_{1}: U_{i} \rightarrow R S:\left\langle I D_{i}, M_{5}, T_{i}\right\rangle:\left\langle M_{1}\right\rangle_{G_{i}}$

Thus

$\mathrm{S} 1: R S \triangleleft\left\langle I D_{i}, M_{5}, T_{i}\right\rangle:\left\langle M_{1}\right\rangle_{G_{i}}$

Applying assumption $\mathrm{ASM}_{4}, \mathrm{~S} 1$ and message meaning rule gives:

$\mathrm{S} 2: R S \mid \equiv U_{i} \sim M_{1}$

According to $A S M_{2}, \mathrm{~S} 2$, freshness-conjuncatenation and nonce verification rule:

S3: $R S \equiv U_{i} \equiv M_{1}$, where information of the parameter $M_{1}$ is used to computed the session key in our protocol.

According to $A S M_{6}, \mathrm{~S} 3$ and jurisdiction rule:

$\mathrm{S} 4: R S \equiv M_{1}$

According to $A S M_{2}, \mathrm{~S} 3$ and session key rule:

$\mathrm{S} 5: R S \equiv R S \stackrel{S K}{\longrightarrow} U_{i}$

(Goal 3)

According to $A \mathrm{SM}_{2}, \mathrm{~S} 5$ and nonce verification rule:

S6: $R S \equiv U_{i} \equiv R S \stackrel{S K}{\longrightarrow} U_{i}$

(Goal 4)

$M S G_{2}: R S \rightarrow U_{i}:\left\langle M_{7}\right\rangle:\left\langle r_{j}\right\rangle_{K_{x}}$

According to seeing rule:

S7: $U_{i} \triangleleft:\left\langle M_{7}\right\rangle:\left\langle r_{j}\right\rangle_{K_{x}}$

Applying the assumption $\mathrm{ASM}_{3}, \mathrm{~S} 7$ and message meaning rule:

S8: $U_{i} \equiv R S \sim r_{j}$

According to $A S M_{1}, \mathrm{~S} 8$, freshness-conjuncatenation and nonce verification rule:

S9: $U_{i} \equiv R S \equiv r_{j}$, where information of the parameter $r_{j}$ is used to computed session key in our protocol.

According to $A S M_{5}, \mathrm{~S} 9$ and jurisdiction rule:

$\mathrm{S} 10: U_{i} \equiv r_{j}$

According to $A S M_{1}, \mathrm{~S} 9$ and session key rule:

S11: $U_{i} \equiv U_{i} \stackrel{S K}{\longrightarrow} R S$

(Goal 1)

According to $A S M_{1}, \mathrm{~S} 11$ and nonce verification rule:

S12: $U_{i} \equiv R S \mid U_{i} \stackrel{S K}{\longrightarrow} R S$

(Goal 2)

The above justification claims that the declared goals have been successfully proven using BAN logic model. Therefore, it can be claimed that the proposed protocol successfully provides mutual authentication property as well as session key negotiation between the user and RS. 


\section{B. Further Security Analysis}

It has been observed that numerous authentication protocols [1], [2], [13], [14], [17], [20] analyze the resilience against known attacks through informal security analysis [21], [22]. Therefore, this section provides the description of the resilience against the known security attacks, such as off-line password guessing attack, privileged insider attack, user impersonation attack, server impersonation attack, known key security attack, stolen-verifier attack, DoS attack and mutual authentication.

\section{1) Off-line password guessing attack}

During the registration phase, $U_{i}$ 's password $P W_{i}$ was never transmitted to RS in plaintext form and the computation of $P W B_{i}$ depends on $P W_{i}$ and random number $b_{i}$. Therefore, if the adversary wants to guess $P W_{i}$, they have to first know $P W B_{i}$, which is used to compute $M_{5}$ in Step 2 of mutual authentication and session key netiation where $M_{5}=E_{K_{x}}\left(M_{1}\left\|M_{4}\right\| P W B_{i} \| C_{i}\right)$ and $P W B_{i}$ is encrypted with key $K_{x}$. Thus, the adversary cannot retrieve $P W B_{i}$ without $K_{x}$. Accordingly, the adversary cannot compute $P W B_{i}$ using $M_{6}$ without $K_{y}$, where $M_{6}=h\left(I D_{i}\left\|P W B_{i}\right\| K_{x}^{\prime}\left\|r_{j}^{\prime}\right\| T_{j}\right)$. Hence, this proposed protocol claims that it is immune to the password guessing attack.

\section{2) Privileged insider attack}

During the registration, as mentioned in the literature [5], [6], a user's password should not be sent to RS in plaintext form during the registration phase in order to resist the insider attack. In the registration phase of this work, $U_{i}$ sends a masked password $P W B_{i}$ to RS instead of $P W_{i}$, where $P W B_{i}=h\left(P W_{i} \| b_{i}\right)$. Therefore, the insider attach of RS cannot extract $P W_{i}$ from $P W B_{i}$ due to the strong collision resistance property of the hash function $h()$.

\section{3) User impersonation attack}

Suppose that an adversary endeavors to impersonate $U_{i}$. In order to do it, the adversary first captures $U_{i}$ 's message from the public channel and then makes an effort to generate another valid message, which should be authenticated by RS. The adversary traps $\left\langle I D_{i}, M_{5}, T_{i}\right\rangle$ from the public channel and tries to compute $\left\langle M_{2}, K_{y}, C_{i}\right\rangle$ using the known information. However, the adversary cannot compute $M_{2}$ and $K_{y}$ without $\theta_{i}$ and $x$, respectively, where $x$ is the secret key of RS. In addition, $C_{i}$ is also secure being stored in the registration software in $U_{i}$ in encrypted form. Therefore, it is difficult task for the adversary to impersonate $U_{i}$.

\section{4) Server impersonation attack}

An adversary may try to impersonate RS in the mutual authentication phase. In this proposes protocol, RS sends $\left\langle M_{7}\right\rangle$ to the registration software in $U_{i}$ through an open channel, where $M_{7}=E_{K_{y}^{\prime}}\left(M_{6} \| r_{j}\right)$. Note that $\left\langle M_{7}\right\rangle$ is encrypted with key $K_{y}$ and it is depends on $M_{6}$ and $r_{j}$, where $M_{6}=h\left(I D_{i}\left\|P W B_{i}\right\| K_{x}^{\prime}\left\|r_{j}\right\| T_{j}\right)$. It is clear that the adversary can easily generate a random number, but to compute $M_{6}$, the adversary needs $\left(P W B_{i}, K_{x}\right)$. However, the adversary is unable to successfully compute $\left(P W B_{i}, K_{x}\right)$ from the public message. Therefore, this proposed protocol can withstand the server impersonation attack.

\section{5) Stolen-verifier attack}

This type of attack occurs when the stored information in RS is leaked, however, the authentication system should not be affected by the adversary. Suppose that the information stored in the table available to RS has been compromised, where the table contains the entries of the form $\left(I D_{i}, U N S I D_{i}, S L_{i},\left(S K_{i} \oplus h\left(I D_{i} \oplus x\right)\right)\right)$. Note that the adversary cannot extract $h\left(I D_{i} \oplus x\right)$ without $S K_{i}$. Furthermore, a valid user is not able to obtain long-term information from RS. Therefore, the adversary is unable to get any advantage after obtaining the stored table. 


\section{6) Denial-of-service attack}

In biometric based authentication, the biometric information may be affected due to noise during the biometric acquisition, resulting in difficulty in reproducing the exact biometric data signature accurately each time. The hash function is very sensitive to even slight changes in the input. Therefore, the hash function cannot be applied directly to the biometric data. A legal user may even fail to login to the remote server due to noisy biometric sensor data. If a biometric based authentication protocol relies on verifying $h\left(B T_{i}^{*}\right)=? h B T_{i}$, in each session, then $U_{i}$ may get rejected and in biometric authentication this phenomenon is called the DoS attack. In order to resist such kind of problem, a fuzzy extractor is typically used. Therefore, the registration software in $U_{i}$ passes the biometric verification of $U_{i}$ and thus, it can withstand the DoS attack.

\section{7) Mutual authentication}

Mutual authentication [23] is typically one of the important and enviable property of any client-server authentication protocol. In Step 3 of the mutual authentication phase of this work, RS verifies the authenticity of $U_{i}$ by checking the condition $M_{3}{ }^{\prime}=$ ? $M_{3}$ whereas $U_{i}$ checks $M_{6}{ }^{\prime}=? M_{6}$ in Step 5 to verify the legitimacy of RS. Therefore, this proposed protocol achieves the mutual authentication property.

\section{8) Man-in-the-middle attack}

In this form of attack, the adversary ensnares the public messages and attempts to act as a middle broker between the user and the remote server. In user impersonation attack, the work demonstrated that the adversary cannot generate a forged login message without knowing the user's secret information. For the same reason, the adversary cannot also impersonate the RS. Therefore, this proposed protocol can withstand the man-in-the-middle attack.

\section{PeRformance ANALysis}

This section appraises the performance of the proposed protocol in terms of computation and communication costs with other competitive protocols [7], [9], [10]. This work uses crypto-operations to evaluate the computation cost. The notations and description of the crypto-operations are:

- $T_{e}$ : Time needed to perform exponentiation operation.

- $T_{p m}$ : Time needed to perform elliptic curve point multiplication operation.

- $T_{h}$ : Time needed to perform one-way hash operation.

- $T_{s}$ : Time needed to perform symmetric key encryption/decryption operation.

TABLE II provides computation costs of this proposed protocol compared to the relevant literature [7], [9], [10]. This proposed protocol requires an increased computation cost, however for the considered device, the increase in computation cost is marginal compared to the significantly improved security benefits.

The communication cost of this work compared to the literature [7], [9], [10] was analyzed. It was observed that this proposed protocol has a lower communication cost than the protocols considered in the literature. For comparison purposes, this work assumed that the length of $I D_{i}, P W_{i}$ and $B T_{i}$ are 64 bits of length each. In addition, the message digest of the hash function, ECCpoint multiplication and symmetric key encryption produced 160-bits, 160-bits and 128-bits, respectively. TABLE III presents the communication overhead cost and it can be observed that the proposed protocol is very efficient in terms of the communication cost. 
TABLE II

COMPARISON OF THE COMPUTATIONAL COST OF THIS WORK

COMPARED TO THE LITERATURE

\begin{tabular}{|c|c|c|c|}
\hline & User cost & Server cost & Total cost \\
\hline Yang et al. [7] & $4 T_{e}+3 T_{h}+1 T_{s}$ & $6 T_{e}+2 T_{h}+1 T_{s}$ & $10 T_{e}+5 T_{h}+2 T_{s}$ \\
\hline Lee et al. [9] & $2 T_{p m}+5 T_{h}+1 T_{s}$ & $2 T_{p m}+4 T_{h}+1 T_{s}$ & $4 T_{p m}+9 T_{h}+2 T_{s}$ \\
\hline He et al. [10] & $2 T_{p m}+5 T_{h}+1 T_{s}$ & $2 T_{p m}+4 T_{h}+1 T_{s}$ & $2 T_{p m}+4 T_{h}+1 T_{s}$ \\
\hline Proposed & $3 T_{p m}+5 T_{h}+3 T_{s}$ & $3 T_{p m}+5 T_{h}+2 T_{s}$ & $6 T_{p m}+10 T_{h}+5 T_{s}$ \\
\hline \multicolumn{4}{|c|}{$\begin{array}{c}\text { TABLE III } \\
\text { COMPARISON OF THE COMMUNICATION COST OF THIS WORK } \\
\text { COMPARED TO THE LITERATURE } \\
\end{array}$} \\
\hline & User & Server & Total cost \\
\hline Yang et al. [7] & 4224 & 1312 & 5536 \\
\hline Lee et al. [9] & 480 & 480 & 960 \\
\hline He et al. [10] & 480 & 480 & 960 \\
\hline Proposed & 256 & 256 & 512 \\
\hline
\end{tabular}

\section{CONCLUSION}

The main intention of this paper is to provide security protection on the stored information in the consumer device from the unauthorized access by implementing an authentication protocol. In order to do it, this paper proposes a mutual authentication and key negotiation protocol using elliptic curve cryptography. The security verification of the protocol has been done using BAN logic and the security analysis ensures that the protocol can withstand several relevant security attacks. The protocol is not only efficient in terms of security attacks, but it also achieves high performance in terms of communication cost in comparison with the existing protocols. Moreover, the proposed protocol provides the mutual authentication property between the participants involved and provides a password update facility to registered users. This work enables secure biometric personal storage devices to be configured from an Internet service and maintained throughout the lifetime of the device.

\section{REFERENCES}

[1] L. Lamport, "Password authentication with insecure communication," Communications of the ACM, vol. 24, no. 11, pp. 770-772, Nov. 1981.

[2] M.-S. Hwang, and L.-H. Li, "A new remote user authentication scheme using smart cards," IEEE Trans. Consumer Electron., vol. CE-46, no. 1, pp. 28-30, Feb. 2000.

[3] H.-M. Sun, "An efficient remote use authentication scheme using smart cards," IEEE Trans. Consumer Electron., vol. CE-46, no. 4, pp. 958-961, Nov. 2000.

[4] C.-K. Chan, and L.M. Cheng, "Cryptanalysis of a remote user authentication scheme using smart cards," IEEE Trans. Consumer Electron., vol. CE-46, no. 4, pp. 992-993, Nov. 2000.

[5] R. Amin, and G. P. Biswas, "A secure light weight scheme for user authentication and key agreement in multi-gateway based wireless sensor networks," Ad Hoc Networks, vol. 36, no. 1, pp. 58-80, Jan. 2016.

[6] R. Amin, and G. P. Biswas, "A novel user authentication and key agreement protocol for accessing multi-medical server usable in TMIS," Journal of Medical Systems, vol. 39, no. 3, pp. 1-17, Mar. 2015.

[7] F.-Y. Yang, T.-D. Wu, and S.-H. Chiu, "A secure control protocol for USB mass storage devices," IEEE Trans. Consumer Electron., vol. CE-56, no. 4, pp. 2339-2343, Nov. 2010.

[8] B. Chen, C. Qin, and L. Yu, "A Secure Access Authentication Scheme for Removable Storage Media," Journal of Information \& Computational Science, vol. 9, no. 15, pp. 4353-4363, Nov. 2012.

[9] C. Lee, C. Chen, and P. Wu, "Three-factor control protocol based on elliptic curve cryptosystem for universal serial bus mass storage devices," IET Computers \& Digital Techniques, vol. 7, no. 1, pp. 48-55, Jan. 2013.

[10] D. He, N. Kumar, J.-H. Lee, and R. S. Sherratt, "Enhanced three-factor security protocol for consumer USB mass storage devices," IEEE Trans. Consumer Electron., vol. CE-60, no. 1, pp. 30-37, Feb. 2014. 
[11] D. Giri, R. S. Sherratt, T. Maitra, and R. Amin, "Efficient Biometric and Password Based Mutual Authentication for Consumer USB Mass Storage Devices," IEEE Trans. Consumer Electron., vol. CE-61, no. 4, pp. 491-499, Nov. 2015.

[12] D. Giri, R. S. Sherratt, and T. Maitra, "A novel and efficient session spanning biometric and password based three-factor authentication protocol for consumer USB mass storage devices," IEEE Trans. Consumer Electron., vol. CE-62, no. 3, pp. 283-291, Aug. 2016.

[13] Y. Dodis, L. Reyzin, and A. Smith, "Fuzzy extractors: How to generate strong keys from biometrics and other noisy data," LNCS, vol. 3027, pp. 523-540, 2004.

[14] X. Boyen, "Reusable cryptographic fuzzy extractors," in Proc. ACM CCS, 2004, pp. 82-91.

[15] R. Amin, and G.P. Biswas, "Anonymity preserving secure hash function based authentication scheme for consumer USB mass storage device," in Proc. IEEE CCCIT, 2015, pp. 1-6.

[16] N. Koblitz, "Elliptic curve cryptosystem," Mathematics of Computation., vol. 48, no. 177, pp. 203-209, Jan. 1987.

[17] V. S. Miller, "Use of elliptic curves in cryptography," LNCS, vol. 218, pp. 417-426, Dec. 2000.

[18] M. Burrows, M. Abadi, and R. Needham, "A logic of authentication," ACM Trans. Computer Systems, vol. 8, no. 1, pp. 18-36, Feb. 1990.

[19] D. He, N. Kumar and N. Chilamkurti, "A secure temporal-credential-based mutual authentication and key agreement scheme with pseudo identity for wireless sensor networks," Information Sciences, vol. 321, pp. 263-277, Nov. 2015.

[20] P. Sarkar, "A simple and generic construction of authenticated encryption with associated data," ACM Trans. Information and System Security, vol. 13, no. 4, pp. 1-16, Dec. 2010.

[21] W.-C. Ku, and S.-M. Chen, "Weaknesses and improvements of an efficient password based remote user authentication scheme using smart cards," IEEE Trans. Consumer Electron., vol. CE-50, no. 1, pp. 204-207, Feb. 2004.

[22] E.-J. Yoon, E.-K. Ryu, and K.-Y. Yoo, "Further improvement of an efficient password based remote user authentication scheme using smart cards," IEEE Trans. Consumer Electron., vol. CE-50, no. 2, pp. 612-614, May. 2004.

[23] S. H. Islam, and G. P. Biswas, "A more efficient and secure ID-based remote mutual authentication with key agreement scheme for mobile devices on elliptic curve cryptosystem," J. Systems and Software, vol. 84, no. 11, pp. 1892-1898, Nov. 2011.

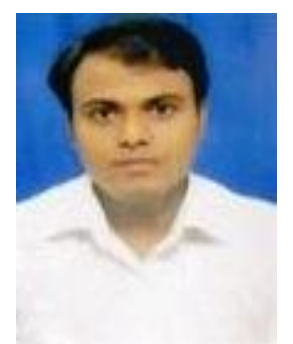

Ruhul Amin received his B.Tech and M.Tech from West Bengal University of Computer Science and Engineering, Indian Engineering in 2009 and 2013, respectively. He was a Ph.D. research scholar in Computer Science and Engineering, Indian School of Mines (ISM), Dhanbad, India. He is currently a Lecturer in the Department of Computer Science and Engineering, Thapar University, Patiala, Punjab, India. He has published many research papers in Journals and Conference proceedings of International reputes. His current research interests include cryptographic authentication protocols and security in wireless sensor networks.

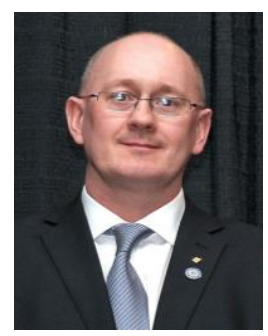

R. Simon Sherratt (M'97-SM'02-F'12) received the B.Eng. degree in Electronic Systems and Control Engineering from Sheffield City Polytechnic, UK in 1992, M.Sc. in Data Telecommunications in 1994 and Ph.D. in video signal processing in 1996 from the University of Salford, UK.

In 1996, he has appointed as a Lecturer in Electronic Engineering at the University of Reading where he is now Professor of Biosensors. His research topic is signal processing and personal communications in consumer devices focusing on wearable devices and healthcare.

He received the $1^{\text {st }}$ place IEEE Chester Sall Memorial Award in 2006, the $2^{\text {nd }}$ place in 2016 and the $3^{\text {rd }}$ place in 2017. He is a reviewer for the IEEE SENSORS JOURNAL and is currently a Senior Editor and Emititus Editor-in-Chief of the IEEE TRANSACTIONS ON CONSUMER ELECTRONICS.

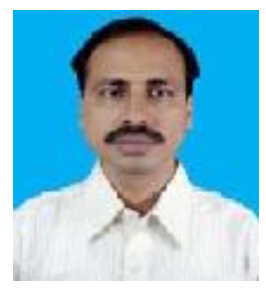

Debasis Giri received the Ph.D degree from the Indian Institute of Technology, Kharagpur, India in 2009. He did his masters (M.Tech and M.Sc) both from Indian Institute of Technology, Kharagpur, India in 2001 and 1998 respectively. Presently he is a Dean under the school of Electronic, Computer Science and Informatics of Haldia Institute of Technology, India, and Professor in the Department of Computer Science and Engineering, Haldia Institute of Technology, India. He has tenth All India Rank with percentile score 98.42 in the Graduate Aptitude Test in Engineering (GATE) Examination in 1999. His current research interests include Cryptography, Network security, Data Hiding, Security in Wireless Sensor Networks and

Security in VANETs.

Dr. Giri is an Editorial Board Member and a Reviewer of many reputed International Journals. Presently he is an Associate Editor of the Journal of Security and Communication Networks (Wiley), the Journal of Communication Systems (Wiley) and the Journal of Electrical and Computer Engineering Innovations. He is also a Program Committee member for many International Conferences. 


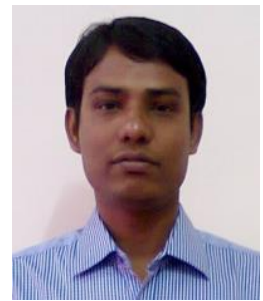

SK Hafizul Islam received the M.Tech from ISM Dhanbad in 2009 and the Ph.D in Computer Science and Engineering from Indian School of Mines, Dhanbad (SM Dhanbad), India. He was an Assistant Professor in the Department of CSIS, BITS Pilani, Pilani Campus, Rajasthan, India and is currently an Assistant Professor in the Department of CSE, Indian Institute of Information Technology, Kalyani (IIIT Kalyani), West Bengal, India. He has published 50 research papers in reputed international Journals and Conference proceedings. He is an Associate Editor of the International journal of Communication Systems, Wiley. His research interest includes Cryptography and Information Security.

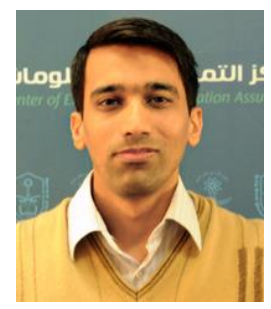

Muhammad Khurram Khan (M'07, SM'12) is currently working as a Full Professor at the Center of Excellence in Information Assurance (CoEIA), King Saud University, Kingdom of Saudi Arabia. He has published over 250 research papers in the journals and conferences of international repute. In addition, he is an inventor of $10 \mathrm{US} / \mathrm{PCT}$ patents.

Prof. Khan is the Editor-in-Chief of Telecommunication Systems Journal, Springer. He is a Fellow of the IET, Fellow of the BCS, Fellow of the FTRA, a member of the IEEE Technical Committee on Security \& Privacy, a member of the IEEE Cybersecurity community, and a member of IEEE Consumer Electronics society. 